\title{
Optimally sparse shearlet approximations of 3D data
}

\author{
Demetrio Labate ${ }^{a}$ and Kanghui Guo ${ }^{b}$ \\ ${ }^{a}$ Department of Mathematics, University of Houston, Houston, Texas 77204; \\ ${ }^{b}$ Department of Mathematics, Missouri State University, Springfield, Missouri 65804
}

\begin{abstract}
Sparse representations of multidimensional data have gained more and more prominence in recent years, in response to the need to process large and multi-dimensional data sets arising from a variety of applications in a timely and effective manner. This is especially important in applications such as remote sensing, satellite imagery, scientific simulations and electronic surveillance. Directional multiscale systems such as shearlets are able to provide sparse representations thanks to their ability to approximate anisotropic features much more efficiently than traditional multiscale representations. In this paper, we show that the shearlet approach is essentially optimal in representing a large class of 3D containing discontinuities along surfaces. This is the first nonadaptive approach to achieve provably optimal sparsity properties in the 3D setting.
\end{abstract}

Keywords: Affine systems, curvelets, shearlets, sparsity, wavelets.

\section{INTRODUCTION}

During the last five years, starting with the introduction of curvelets ${ }^{2}$ and shearlets, ${ }^{14}$ a new class of 2 -dimensional representations have emerged which combine multiscale analysis with the ability to efficiently capture anisotropic and directional information. These new representations go far beyond traditional wavelets with respect to their approximation properties, since they can deal much more effectively with edges and other distributed singularities which are dominant features in typical images. Indeed, even though curvelets and shearlets are based on very different mathematical constructions, both systems form a tight frame of well localized waveforms defined not only at various scales and locations, like wavelets, but also at various orientations, and with highly anisotropic shapes. Thanks to their properties, both methods were found to provide essentially optimally sparse representations for a large class of 2-dimensional data, ${ }^{2,9}$ hence outperforming standard wavelet approximations.

Specifically, consider the class $E$ of cartoon-like images, defined as the collection of bounded functions on $[0,1]^{2}$ which are $C^{2}$ regular, up to discontinuities along $C^{2}$ curves (see Ref.[9] for the precise definition). For any $f \in E$, let $f_{N}^{S}$ be the corresponding $N$-term approximation obtained by taking the $N$ largest coefficients in the shearlet (or curvelet) expansion. Then the approximation error satisfies the asymptotic decay rate

$$
\left\|f-f_{N}^{S}\right\|_{2}^{2} \asymp N^{-2}(\log N)^{3}, \quad \text { as } N \rightarrow \infty,
$$

which is essentially the optimal error rate. Indeed, one can prove ${ }^{4}$ that no orthonormal bases or tight frames can yield approximation error rates that are better than $N^{-2}$. In particular, this shows that shearlet and curvelet approximations greatly improve wavelet approximations whose error rate is only $O\left(N^{-1}\right)$.

Due to their excellent approximation properties, shearlets and curvelets have been successfully employed in a number of signal and image processing applications including image denoising, edge detection and geometric separation. ${ }^{3,5,7,8,18,22}$ However, all this research was so far focused essentially on the 2-dimensional setting. While it has been suggested in several papers that the general ideas of this approach can be extended to higher dimensions, and even 3-dimensional versions of the corresponding numerical codes have been introduced, ${ }^{1}$ no proper 3D theory was developed until very recently. In particular, no results about the approximation properties of $3 \mathrm{D}$ shearlets or curvelets systems were known so far. It is important to notice that the extension of the approximation estimate (1) to the 3D setting is highly nontrivial, since the arguments used in the 2D case do not extend directly to higher dimensions. In addition, it must be recalled that there is not a unique way to extend

D.L. E-mail: dlabate@math.u.edu; K.G.E-mail: KanghuiGuo@MissouriState.edu 
the construction of shearlets or curvelets to dimensions larger than 2, due to the increasing complexity of the geometry in higher dimensions.

In this paper, we describe a number of very recent results, illustrating the extension of the shearlet approach to 3D. Specifically, we show that the shearlet construction extends naturally to the 3-dimensional setting and this approach yields optimally sparse nonadaptive representations of 3D data. In fact, we will construct a Parseval frame of shearlets to represent 3-dimensional functions $f$ which are smooth away from discontinuities along $C^{2}$ boundaries, and show that the $N$-term approximation $f_{N}^{S}$, obtained from the $N$ largest coefficients of its shearlet representation, satisfies the estimate:

$$
\left\|f-f_{N}^{S}\right\|_{2}^{2} \asymp N^{-1}(\log N)^{2}, \quad \text { as } N \rightarrow \infty .
$$

Similar to the corresponding $2 \mathrm{D}$ estimate, this is essentially the optimal approximation rate for this type of functions, ${ }^{4}$ in the sense that no orthonormal bases or Parseval frames can yield approximation rates that are better than $N^{-1}$. In contrast, more traditional methods based on wavelet and Fourier approximations are significantly less efficient since their asymptotic approximation rate only decays as $N^{-1 / 2}$ and $N^{-1 / 3}$, respectively. ${ }^{6,19}$ This result was first announced by the authors in Ref.[11] and is the first and, at present, the only nonadaptive construction which is provably optimal (up to a loglike factor) for a large class of 3D data.

Remark. Very recently, a similar (essentially) optimal sparsity result was announced by Kutyniok, Lemvig and Lim, which is based on a new construction of compactly supported shearlet frames ${ }^{17}$ (see also [16] for the construction of compactly supported shearlet frames in the $2 \mathrm{D}$ case). No proof of this result is available at the moment. Notice that the structure of this new system of shearlets is very different from the one which is discussed in this paper.

\section{SHEARLETS IN 3D}

The shearlet approach is derived from the framework of wavelets with composite dilations, originally introduced by the authors and their collaborators, ${ }^{14,15}$ which defines collections of functions in $L^{2}\left(\mathbb{R}^{n}\right)$ of the form

$$
\left\{\psi_{j, \ell, k}(x)=|\operatorname{det} A|^{j / 2} \psi\left(B_{\ell} A^{j} x-k\right): j \in \mathbb{Z}, \ell \in L, k \in \mathbb{Z}^{n}\right\}
$$

where $\psi \in L^{2}\left(\mathbb{R}^{n}\right), A$ is an expanding invertible $n \times n$ matrix, $B$ is matrix for which $|\operatorname{det} B|=1$ and $L$ is a countable indexing set. This setting provides a general method for the construction of function systems made up of functions ranging not only at various scales (associated with the powers of the dilation matrix $A$ ) and locations, but also according to various orthogonal transformations controlled by the matrices $\left\{B_{\ell}\right\}$. For example, the matrices $\left\{B_{\ell}\right\}$ can be associated with discrete rotations, or with shearing transformations, as in the case of shearlets. One major feature of this approach is that all the elements of the function systems are obtained from the action of a countable collection of unitary operators on a single (or possibly finite) generator function, and this offers several advantages in terms of mathematical flexibility, ability to naturally generalize to higher dimensions and close connection with the classical theory of affine systems. This is a major difference with respect to the curvelet approach, which is not of this type.

\subsection{Shearlet Systems in 3D}

In dimension $n=2$, a shearlet system* is obtained from $(3)$ by choosing $A=\left(\begin{array}{ll}4 & 0 \\ 0 & 2\end{array}\right)$ and $B_{\ell}=\left(\begin{array}{ll}1 & \ell \\ 0 & 1\end{array}\right), \ell \in \mathbb{Z}$. Notice that the matrices $A^{j}, j \in \mathbb{Z}$, produce parabolic scaling, that is, the dilation factor along one coordinate axis is quadratic with respect to the orthogonal direction. The matrices $B_{\ell}, \ell \in \mathbb{Z}$, on the other hand, are nonexpanding and are called shearing matrices due to the particular orthogonal geometric action to which they are associated. ${ }^{15}$ As mentioned above and proved by the authors in a previous work, ${ }^{9}$ for an appropriate generating function $\psi \in L^{2}\left(\mathbb{R}^{2}\right)$, the corresponding $2 \mathrm{D}$ shearlet system forms a Parseval frame of $L^{2}\left(\mathbb{R}^{2}\right)$ which provides nearly optimal approximation properties for cartoon-like images as in the estimate (1). Notice that the parabolic scaling plays a critical role in the proof of this result.

\footnotetext{
${ }^{*}$ We are referring here to the shearlet system on the horizontal cone, that is, one with frequency support in the region

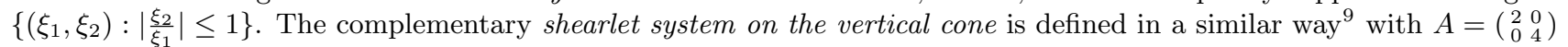
and $B_{\ell}=\left(\begin{array}{cc}1 & 0 \\ \ell & 1\end{array}\right)$.
} 
To extend the shearlet systems to $n=3$, there is more than one options which is consistent with the $2 \mathrm{D}$ construction. In particular, it was already observed in an early paper ${ }^{15}$ that $3 \times 3$ shearlet matrices can be defined as

$$
B_{\ell}=\left(\begin{array}{ccc}
1 & \ell_{1} & \ell_{2} \\
0 & 1 & \ell_{3} \\
0 & 0 & 1
\end{array}\right), \quad \ell=\left(\ell_{1}, \ell_{2}, \ell_{3}\right) \in \mathbb{Z}^{3}
$$

or as

$$
B_{\ell}^{(1)}=\left(\begin{array}{ccc}
1 & \ell_{1} & \ell_{2} \\
0 & 1 & 0 \\
0 & 0 & 1
\end{array}\right), \quad \ell=\left(\ell_{1}, \ell_{2}\right) \in \mathbb{Z}^{2}
$$

In the following, we will consider the second choice, since it simplifies the construction of the generator function. For the dilation matrix, there are two ways to extend the parabolic scaling matrix to 3D, namely, one can choose

$$
A=\left(\begin{array}{lll}
4 & 0 & 0 \\
0 & 4 & 0 \\
0 & 0 & 2
\end{array}\right) \quad \text { or } \quad A_{(1)}=\left(\begin{array}{lll}
4 & 0 & 0 \\
0 & 2 & 0 \\
0 & 0 & 2
\end{array}\right)
$$

As will be shown below, the choice of dilation matrix $A_{(1)}$ leads to a system of wafer-like waveforms, which is well suited to approximate surface singularities. On the other hand, the second choice of dilation matrices leads to a system of needle-like waveforms, which appears to be better suited to capture curvilinear singularities. It turns out that the first choice is the correct one if one wants to achieve essentially optimal approximations of piecewise smooth 3D data. Hence, for a fixed shearlet generator function $\psi \in L^{2}\left(\mathbb{R}^{3}\right)$, we define a $3 D$ shearlet system as a collection of functions of the form

$$
\left\{\psi_{j, \ell, k}(x)=\left|\operatorname{det} A_{(1)}\right|^{j / 2} \psi\left(B_{\ell}^{(1)} A_{(1)}^{j} x-k\right): j \in \mathbb{Z}, \ell \in L \subset \mathbb{Z}^{2}, k \in \mathbb{Z}^{3}\right\},
$$

where the shear matrices $B_{\ell}^{(1)}$ are given by (4) and the matrix $A_{(1)}$ is given by (5).

Let us now show how to construct a shearlet generator $\psi$ such that the corresponding 3D shearlet system is a well localized Parseval frame of shearlets. As usual, it is convenient to construct the system of shearlets in the Fourier domain. In the following, we will adopt the following definition of the Fourier transform of a function $f \in L^{2}\left(\mathbb{R}^{3}\right)$ :

$$
\hat{f}(\xi)=\int_{\mathbb{R}^{3}} f(x) e^{-2 \pi i \xi x} d x
$$

where we assume that the vectors $x \in \mathbb{R}^{3}$ (the space domain) are column vectors, whereas the vectors $\xi \in \widehat{\mathbb{R}}^{3}$ (the Fourier domain) are row vectors. It follows that, if $M$ is a $3 \times 3$ invertible matrix and $j \in \mathbb{Z}$, the Fourier transform of

$$
f_{j}(x)=|\operatorname{det} M|^{j / 2} f\left(M^{j} x\right)
$$

is the function

$$
\hat{f}_{j}(\xi)=|\operatorname{det} M|^{-j / 2} f\left(\xi M^{-j}\right) .
$$

We can now state and prove the following useful result.

Theorem 2.1. For $\xi=\left(\xi_{1}, \xi_{2}, \xi_{3}\right) \in \widehat{\mathbb{R}}^{3}$, let $\psi^{(1)}$ be defined by

$$
\hat{\psi}^{(1)}(\xi)=\hat{\psi}_{1}\left(\xi_{1}\right) \hat{\psi}_{2}\left(\frac{\xi_{2}}{\xi_{1}}\right) \hat{\psi}_{2}\left(\frac{\xi_{3}}{\xi_{1}}\right)
$$

where $\psi_{1}$ and $\psi_{2}$ satisfy the following assumptions:

(i) $\hat{\psi}_{1} \in C^{\infty}(\widehat{\mathbb{R}})$, supp $\hat{\psi}_{1} \subset\left[-\frac{1}{2},-\frac{1}{16}\right] \cup\left[\frac{1}{16}, \frac{1}{2}\right]$ and

$$
\sum_{j \geq 0}\left|\hat{\psi}_{1}\left(2^{-2 j} \omega\right)\right|^{2}=1 \quad \text { for }|\omega| \geq \frac{1}{8}
$$




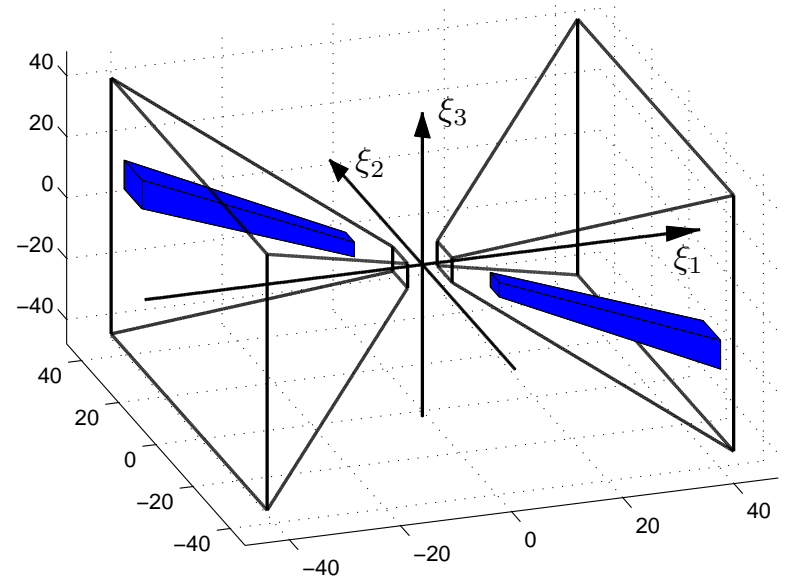

Figure 1. The figure illustrates the frequency support of a typical element of the shearlet system (6). Notice that these elements are contained inside the pyramidal region $\mathcal{D}_{C_{1}}$ defined in Theorem 2.1.

(ii) $\hat{\psi}_{2} \in C^{\infty}(\widehat{\mathbb{R}})$, supp $\hat{\psi}_{2} \subset[-1,1]$ and

$$
\left|\hat{\psi}_{2}(\omega-1)\right|^{2}+\left|\hat{\psi}_{2}(\omega)\right|^{2}+\left|\hat{\psi}_{2}(\omega+1)\right|^{2}=1 \quad \text { for }|\omega| \leq 1 .
$$

Then the $3 D$ shearlet system (6), where $\psi=\psi^{(1)}$ and $L=\left\{\left(\ell_{1}, \ell_{2}\right):\left|\ell_{1}\right|,\left|\ell_{2}\right| \leq 2^{j}\right\}$, is a Parseval frame for $L^{2}\left(\mathcal{D}_{C_{1}}\right)^{\vee}=\left\{f \in L^{2}\left(\mathbb{R}^{3}\right): \operatorname{supp} \hat{f} \subset \mathcal{D}_{C_{1}}\right\}$, where $\mathcal{D}_{C_{1}}=\left\{\left(\xi_{1}, \xi_{2}, \xi_{3}\right) \in \widehat{\mathbb{R}}^{3}:\left|\xi_{1}\right| \geq \frac{1}{8},\left|\frac{\xi_{2}}{\xi_{1}}\right| \leq 1,\left|\frac{\xi_{3}}{\xi_{1}}\right| \leq 1\right\}$.

Proof. It follows from equation (8) that, for any $j \geq 0$,

$$
\sum_{m=-2^{j}}^{2^{j}}\left|\hat{\psi}_{2}\left(2^{j} \omega+m\right)\right|^{2}=1, \quad \text { for }|\omega| \leq 1 .
$$

By direct computation, in the frequency domain, the elements of the shearlet system (6) have the form

$$
\hat{\psi}_{j, \ell, k}^{(1)}(\xi)=\left|\operatorname{det} A_{(1)}\right|^{-j / 2} \psi^{(1)}\left(\xi A_{(1)}^{-j} B_{-\ell}^{(1)}\right) e^{2 \pi i \xi A_{(1)}^{-j} B_{-\ell}^{(1)} k} .
$$

Hence, using equations (7), (9) and the observation that

$$
\left(\xi_{1}, \xi_{2}, \xi_{3}\right) A_{(1)}^{-j} B_{-\ell}^{(1)}=\left(2^{-2 j} \xi_{1},-\ell_{1} 2^{-2 j} \xi_{1}+2^{-j} \xi_{2},-\ell_{2} 2^{-2 j} \xi_{1}+2^{-j} \xi_{3}\right),
$$

a direct computation gives that:

$$
\begin{aligned}
\sum_{j \geq 0} \sum_{\ell_{1}=-2^{j}}^{2^{j}} \sum_{\ell_{2}=-2^{j}}^{2^{j}}\left|\hat{\psi}^{(1)}\left(\xi A_{(1)}^{-j} B_{-\ell}^{(1)}\right)\right|^{2} & =\sum_{j \geq 0} \sum_{\ell_{1}=-2^{j}}^{2^{j}} \sum_{\ell_{2}=-2^{j}}^{2^{j}}\left|\hat{\psi}_{1}\left(2^{-2 j} \xi_{1}\right)\right|^{2}\left|\hat{\psi}_{2}\left(2^{j} \frac{\xi_{2}}{\xi_{1}}-\ell_{1}\right)\right|^{2}\left|\hat{\psi}_{2}\left(2^{j} \frac{\xi_{3}}{\xi_{1}}-\ell_{2}\right)\right|^{2} \\
& =\sum_{j \geq 0}\left|\hat{\psi}_{1}\left(2^{-2 j} \xi_{1}\right)\right|^{2} \sum_{\ell_{1}=-2^{j}}^{2^{j}}\left|\hat{\psi}_{2}\left(2^{j} \frac{\xi_{2}}{\xi_{1}}-\ell_{1}\right)\right|^{2} \sum_{\ell_{2}=-2^{j}}^{2^{j}}\left|\hat{\psi}_{2}\left(2^{j} \frac{\xi_{3}}{\xi_{1}}-\ell_{2}\right)\right|^{2}=1,
\end{aligned}
$$

for all $\left(\xi_{1}, \xi_{2}, \xi_{3}\right) \in \mathcal{D}_{C_{1}}$. This equality, together with the fact that $\hat{\psi}^{(1)}$ is supported inside $\left[-\frac{1}{2}, \frac{1}{2}\right]^{3}$, implies that the shearlet system $(6)$, where $\psi=\psi^{(1)}$ and $L=\left\{\left(\ell_{1}, \ell_{2}\right):\left|\ell_{1}\right|,\left|\ell_{2}\right| \leq 2^{j}\right\}$, is a Parseval frame for $L^{2}\left(\mathcal{D}_{C_{1}}\right)^{\vee}$. 

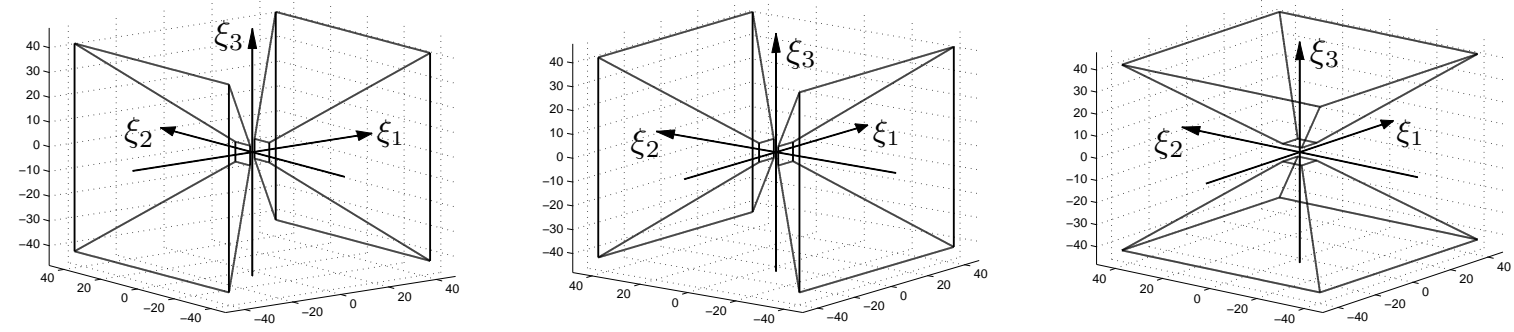

Figure 2. From left to right, the figure illustrates the pyramidal regions $\mathcal{D}_{C_{1}}, \mathcal{D}_{C_{2}}$ and $\mathcal{D}_{C_{3}}$ in the frequency space $\widehat{\mathbb{R}}^{3}$.

As shown in Ref.[9], there are several examples of functions $\psi_{1}, \psi_{2}$ satisfying the assumptions of Theorem 2.1. Similar to the corresponding $2 \mathrm{D}$ construction, ${ }^{9}$ the result shows that the shearlet elements $\left\{\psi_{j, \ell, k}^{(1)}\right\}$ are welllocalized waveforms since each $\hat{\psi}_{j, \ell, k}^{(1)} \in C_{0}^{\infty}\left(\widehat{\mathbb{R}}^{3}\right)$. The functions $\left\{\psi_{j, \ell, k}^{(1)}\right\}$ are ranging over countably many scales (associated with $j \in \mathbb{Z}$ ), countably many orientations (associated with the two-dimensional indices $\ell=\left(\ell_{1}, \ell_{2}\right) \in$ $\mathbb{Z}^{2}$ ) and countably many spatial locations (associated $k \in \mathbb{Z}^{3}$ ). Notice that, for each fixed $j \in \mathbb{Z}$, the frequency support of each $\psi_{j, \ell, k}^{(1)}$ is contained inside a parallelepiped of approximate size $2^{2 j} \times 2^{j} \times 2^{j}$. Hence these support regions become increasingly more elongated at finer scales (See Figure 1).

The construction presented above provides a Parseval frame only for the subspace $L^{2}\left(\mathcal{D}_{C_{1}}\right)^{\vee}$ of functions in $L^{2}$ whose frequency support is contained in the pyramidal region $\mathcal{D}_{C_{1}}$. To obtain a Parseval frame for the larger space $L^{2}\left(\mathbb{R}^{3}\right)$, one can similarly construct a second Parseval frame of shearlets with frequency support in the pyramidal region $\mathcal{D}_{C_{2}}=\left\{\left(\xi_{1}, \xi_{2}, \xi_{3}\right) \in \widehat{\mathbb{R}}^{3}:\left|\xi_{2}\right| \geq \frac{1}{8},\left|\frac{\xi_{1}}{\xi_{2}}\right| \leq 1,\left|\frac{\xi_{3}}{\xi_{2}}\right| \leq 1\right\}$, and a third Parseval frame of shearlets with frequency support in the pyramidal region $\mathcal{D}_{C_{3}}=\left\{\left(\xi_{1}, \xi_{2}, \xi_{3}\right) \in \widehat{\mathbb{R}}^{3}:\left|\xi_{3}\right| \geq \frac{1}{8},\left|\frac{\xi_{1}}{\xi_{3}}\right| \leq 1,\left|\frac{\xi_{2}}{\xi_{3}}\right| \leq 1\right\}$ (see Figure 2). Specifically, for $\xi=\left(\xi_{1}, \xi_{2}, \xi_{3}\right) \in \widehat{\mathbb{R}}^{3}$, let $\psi^{(2)}, \psi^{(3)}$ be defined by

$$
\hat{\psi}^{(2)}(\xi)=\hat{\psi}_{1}\left(\xi_{2}\right) \hat{\psi}_{2}\left(\frac{\xi_{1}}{\xi_{2}}\right) \hat{\psi}_{2}\left(\frac{\xi_{3}}{\xi_{2}}\right), \quad \hat{\psi}^{(3)}(\xi)=\hat{\psi}_{1}\left(\xi_{3}\right) \hat{\psi}_{2}\left(\frac{\xi_{1}}{\xi_{3}}\right) \hat{\psi}_{2}\left(\frac{\xi_{2}}{\xi_{3}}\right)
$$

where $\psi_{1}$ and $\psi_{2}$ are defined as above. Also, let

$$
A_{(2)}=\left(\begin{array}{lll}
2 & 0 & 0 \\
0 & 4 & 0 \\
0 & 0 & 2
\end{array}\right), \quad A_{(3)}=\left(\begin{array}{lll}
2 & 0 & 0 \\
0 & 2 & 0 \\
0 & 0 & 4
\end{array}\right), \quad B_{\ell}^{(2)}=\left(\begin{array}{ccc}
1 & 0 & 0 \\
\ell_{1} & 1 & \ell_{2} \\
0 & 0 & 1
\end{array}\right), \quad B_{\ell}^{(3)}=\left(\begin{array}{ccc}
1 & 0 & 0 \\
0 & 1 & 0 \\
\ell_{1} & \ell_{2} & 1
\end{array}\right),
$$

where $\ell=\left(\ell_{1}, \ell_{2}, \ell_{3}\right) \in \mathbb{Z}^{3}$. Then, by repeating the argument of Theorem 2.1 , one shows that, for $i=2,3$, the systems

$$
\left\{\psi_{j, \ell, k}^{(i)}(x)=\left|\operatorname{det} A_{(i)}\right|^{j / 2} \psi^{(i)}\left(B_{\ell}^{(i)} A_{(i)}^{j} x-k\right): j \in \mathbb{Z}, \ell \in L \subset \mathbb{Z}^{2}, k \in \mathbb{Z}^{3}\right\},
$$

where $L=\left\{\left(\ell_{1}, \ell_{2}\right):\left|\ell_{1}\right|,\left|\ell_{2}\right| \leq 2^{j}\right\}$, are Parseval frames for $L^{2}\left(\mathcal{D}_{C_{i}}\right)^{\vee}, i=2,3$. Finally, one can easily construct a Parseval frame of translates $\left\{\phi(\cdot-k): k \in \mathbb{Z}^{3}\right\}$ for $V_{0}=L^{2}\left(\left[-\frac{1}{8}, \frac{1}{8}\right]^{3}\right)^{\vee}$. Specifically, we will choose $\phi$ such that

$$
\begin{aligned}
& |\hat{\phi}(\xi)|^{2}+\sum_{j \geq 0} \sum_{\ell_{1}, \ell_{2}=-2^{j}}^{2^{j}}\left|\hat{\psi}\left(\xi A^{-j} B_{-\ell}\right)\right|^{2} \chi_{\mathcal{D}_{C_{1}}}(\xi)+\sum_{j \geq 0} \sum_{\ell_{1}, \ell_{2}=-2^{j}}^{2^{j}}\left|\hat{\psi}^{(2)}\left(\xi A_{(2)}^{-j} B_{-\ell}^{(2)}\right)\right|^{2} \chi_{\mathcal{D}_{C_{2}}}(\xi) \\
& \quad+\sum_{j \geq 0} \sum_{\ell_{1}, \ell_{2}=-2^{j}}^{2^{j}}\left|\hat{\psi}^{(3)}\left(\xi A_{(3)}^{-j} B_{-\ell}^{(3)}\right)\right|^{2} \chi_{\mathcal{D}_{C_{3}}}(\xi)=1 \quad \text { for a.e. } \xi \in \widehat{\mathbb{R}}^{3} .
\end{aligned}
$$

Thus, using the notation introduced above, we define the pyramid-adapted $3 D$ shearlet system

$$
S\left(\psi^{(1)}, \psi^{(2)}, \psi^{(3)}, \phi\right)=\Psi\left(\psi^{(1)}\right) \cup \widetilde{\Psi}\left(\psi^{(2)}\right) \cup \widetilde{\widetilde{\Psi}}\left(\psi^{(3)}\right) \cup \Phi(\phi),
$$


where

$$
\begin{aligned}
\Psi\left(\psi^{(1)}\right) & =\left\{P_{C_{1}} \psi_{j, \ell, k}^{(1)}: \psi_{j, \ell, k}^{(1)}(x)=\left|\operatorname{det} A_{(1)}\right|^{j / 2} \psi^{(1)}\left(B_{\ell}^{(1)} A_{(1)}^{j} x-k\right): j \in \mathbb{Z}, \ell \in L \subset \mathbb{Z}^{2}, k \in \mathbb{Z}^{3}\right\}, \\
\widetilde{\Psi}\left(\psi^{(2)}\right) & =\left\{P_{C_{2}} \psi_{j, \ell, k}^{(2)}: \psi_{j, \ell, k}^{(2)}(x)=\left|\operatorname{det} A_{(2)}\right|^{j / 2} \psi^{(2)}\left(B_{\ell}^{(2)} A_{(2)}^{j} x-k\right): j \in \mathbb{Z}, \ell \in L \subset \mathbb{Z}^{2}, k \in \mathbb{Z}^{3}\right\}, \\
\widetilde{\Psi}\left(\psi^{(3)}\right) & =\left\{P_{C_{3}} \psi_{j, \ell, k}^{(3)}: \psi_{j, \ell, k}^{(3)}(x)=\left|\operatorname{det} A_{(3)}\right|^{j / 2} \psi^{(3)}\left(B_{\ell}^{(3)} A_{(3)}^{j} x-k\right): j \in \mathbb{Z}, \ell \in L \subset \mathbb{Z}^{2}, k \in \mathbb{Z}^{3}\right\}, \\
\Phi(\phi) & \left.=\{\phi(x-k)):, k \in \mathbb{Z}^{3}\right\},
\end{aligned}
$$

$L=\left\{\left(\ell_{1}, \ell_{2}\right):\left|\ell_{1}\right|,\left|\ell_{2}\right| \leq 2^{j}\right\}$ and $P_{C_{i}}, i=1,2,3$, is the projection operator defined by $\left(P_{C_{i}} g\right)^{\wedge}(\xi)=\hat{g}(\xi) \chi_{\mathcal{D}_{C_{i}}}(\xi)$. Notice that the projection operators $P_{C_{i}}$ do not affect the elements of the shearlet systems inside each pyramidal region $C_{i}$, but only the elements which lie at the boundaries of those pyramidal regions. Specifically, the projection operators have the effect of truncating (in the frequency domain) the "boundary" elements of each pyramidal region so that the four systems which have been used to define $S\left(\psi^{(1)}, \psi^{(2)}, \psi^{(3)}, \phi\right)$ merge nicely (up to negligible sets) to form a Parseval frame of $L^{2}\left(\mathbb{R}^{3}\right)$. Indeed, from the observations above, an argument very similar to Theorem 2.2 shows that:

TheOREM 2.2. The pyramid-adapted $3 D$ shearlet system $S\left(\psi^{(1)}, \psi^{(2)}, \psi^{(3)}, \phi\right)$ is a Parseval frame of $L^{2}\left(\mathbb{R}^{3}\right)$.

\subsection{Optimally Sparse Shearlet Approximations}

Our main motivation for the introduction of the well localized system of 3D shearlets described above is to overcome the limitations of wavelets with respect to their ability to approximate 3D data with discontinuities along surfaces.

To state our result precisely, let us consider the class $\mathcal{E} \subset L^{2}\left(\mathbb{R}^{3}\right)$ of functions which are $C^{2}$ away from a $C^{2}$ surface as the collection of functions of the form

$$
f=f_{0}+f_{1} \chi_{B}
$$

where $f_{0}, f_{1} \in C_{0}^{2}\left([0,1]^{3}\right), B$ is a simple $C^{2}$ surface and $\|f\|_{C^{2}}=\sum_{|\alpha|<2}\left\|D^{\alpha} f\right\|_{\infty} \leq 1$. These functions are the $3 \mathrm{D}$ analogue of the cartoon-like images in 2D and provide a simplified model of typical 3D data sets (e.g., videos of natural scenes).

It is quite known that wavelet approximations are not very efficient in dealing with this type of data since wavelets are unable to approximate the discontinuous surface efficiently. This can be illustrated using the following heuristic argument. Namely, let $f$ be a function in $\mathcal{E}$ containing a discontinuity along a $C^{2}$ surface, and consider the wavelet expansion of $f$ using an orthonormal smooth wavelet basis $\left\{\phi_{j, k}(x)=2^{3 j} \phi\left(2^{2 j} x-k\right)\right.$ : $\left.j \in \mathbb{Z}, k \in \mathbb{Z}^{3}\right\}$ (we choose dilation factors $2^{2 j}$ rather than $2^{j}$ for consistency with the shearlet system which will be considered next). At scale $2^{-2 j}$, a wavelet $\phi_{j, k}(x)$ is essentially supported on a box of size $2^{-2 j} \times 2^{-2 j} \times 2^{-2 j}$. Hence, since the surface of discontinuity has finite area, there are approximately $2^{4 j}$ wavelet coefficients $C_{j, k}(f)=$ $\left\langle f, \phi_{j, k}\right\rangle$ associated with the surface, while the remaining coefficients are negligible at fine scales. Since

$$
\int_{\mathbb{R}^{3}}\left|\phi_{j, k}(x)\right| d x=2^{3 j} \int\left|\psi\left(2^{2 j} x-k\right)\right| d x=2^{-3 j} \int_{\mathbb{R}^{3}}|\phi(y)| d y
$$

a direct computation shows that, at scale $2^{-2 j}$, all these wavelet coefficients are controlled by

$$
\left|C_{j, k}(f)\right| \leq\|f\|_{\infty}\left\|\phi_{j, k}\right\|_{L^{1}} \leq C 2^{-3 j}
$$

If follows that the $N$-th largest wavelet coefficient $\left|C_{N}(f)\right|$ is bounded by $O\left(N^{-3 / 4}\right)$ and, thus, if $f_{N}^{W}$ is the approximation of $f$ obtained by taking the $N$ largest coefficients of its wavelet expansion, the $L^{2}$-error obeys the estimate:

$$
\left\|f-f_{N}^{W}\right\|_{L^{2}}^{2} \leq \sum_{\ell>N}\left|C_{\ell}(f)\right|^{2} \leq C N^{-1 / 2}
$$

Let us now examine, using a similar heuristic argument, the approximation properties of the 3D shearlet system. As we observed above, at scale $2^{-2 j}$, the elements of the shearlet system $\psi_{j, \ell, k}$ are essentially supported 


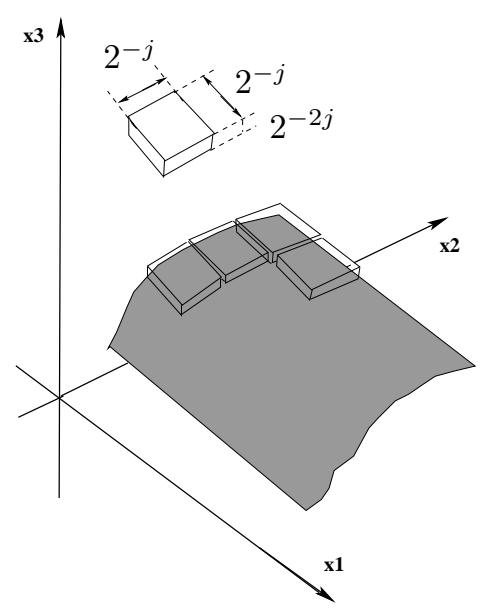

Figure 3. At scale $2^{-2 j}$, each shearlet element $\psi_{j, \ell, k}$ is essentially supported inside a box of size $2^{j} \times 2^{j} \times 2^{-2 j}$. The figure illustrates that, at scale $2^{-2 j}$, there are approximately $2^{j} \times 2^{j}=2^{2 j}$ shearlet elements which are tangent to a surface (of finite area).

on a parallelepiped of size $2^{-2 j} \times 2^{-j} \times 2^{-j}$. At fine scales, due to the properties of the shearlet functions, only the shearlet elements which are tangent to the surface of discontinuity of $f$ will produce significant shearlet coefficients $\left\langle f, \psi_{j, \ell, k}\right\rangle$, and there are about are $2^{2 j}$ coefficients of this type (see illustration in Figure 3 ). Again, a direct computation shows that

$$
\int_{\mathbb{R}^{3}}\left|\psi_{j, \ell, k}(x)\right| d x=2^{-2 j} \int_{\mathbb{R}^{3}}|\psi(y)| d y,
$$

so that, at scale $2^{-2 j}$, all these shearlet coefficients $S_{j, \ell, k}(f)=\left\langle f, \psi_{j, \ell, k}\right\rangle$ are controlled by

$$
\left|S_{j, \ell, k}(f)\right| \leq\|f\|_{\infty}\left\|\psi_{j, \ell, k}\right\|_{L^{1}} \leq C 2^{-2 j} .
$$

It follows that the $N$-th largest shearlet coefficient $\left|S_{N}(f)\right|$ is bounded by $O\left(N^{-1}\right)$ and this implies that, if $f_{N}$ is the approximation of $f$ computed by taking the $N$ largest coefficients of its shearlets expansion, the $L^{2}$-error approximately obeys the estimate:

$$
\left\|f-f_{N}^{S}\right\|_{L^{2}}^{2} \leq \sum_{\ell>N}\left|S_{\ell}(f)\right|^{2} \leq C N^{-1}
$$

It can be proved that this is the optimal approximation rate for this type of functions, ${ }^{4}$ in the sense that no orthonormal bases or Parseval frames can yield approximation rates that are better than $N^{-1}$.

Despite being based on a simple heuristic argument, it turns out that the estimates we have just presented are essentially correct. In fact, we have the following precise result, that was first announced by the authors in Ref.[11].

TheOREm 2.3. Let $\left\{\psi_{\mu}\right\}_{\mu \in M}$, where $M$ is a countable index set, be the Parseval frame of shearlets given by (11). For $f \in \mathcal{E}^{2}$, let

$$
f_{N}^{S}=\sum_{\mu \in I_{N}}\left\langle f, \psi_{\mu}\right\rangle \psi_{\mu}
$$

where $I_{N} \subset M$ is the set of indices corresponding to the $N$ largest entries of the sequence $\left\{\left|\left\langle f, \psi_{\mu}\right\rangle\right|^{2}: \mu \in M\right\}$. Then

$$
\left\|f-f_{N}^{S}\right\|_{2}^{2} \leq C N^{-1}(\log N)^{2} .
$$

Hence the result shows that the shearlet approximations of $f \in \mathcal{E}^{2}$ is optimal up to a log-like factor.

The proof of Theorem 2.3 is rather involved and its detailed description goes beyond the scope of this paper. However, it is important to remark that the proof relies on the regularity and localization properties of the 
sharlet system and the choice of the parabolic scaling. With respect to the $2 \mathrm{D}$ argument, the main technical novelty in the proof consists in the use of the Ray Transform (see Ref.[20]) to prove a fundamental estimate for the decay of the shearlet coefficients corresponding to the surfaces of discontinuity. Specifically, let us assume that, at a sufficiently fine scale, the surface of discontinuity is parametrized as:

$$
x_{1}=E\left(x_{2}, x_{3}\right), \quad-2^{-j} \leq x_{2}, x_{3} \leq 2^{-j} .
$$

Define a surface fragment as the following localized version of $f$ :

$$
f_{l o c}(x)=w_{0}\left(2^{j} x\right) g(x) \chi_{\left[x_{1}>E\left(x_{2}, x_{3}\right)\right]}(x), \quad x \in\left[-2^{-j}, 2^{-j}\right]^{3},
$$

where $w_{0}$ is a nonnegative $C^{\infty}$ window function with support in $[-1,1]^{3}$. Then we obtain the following fundamental estimate valid for the shearlet system (6) defined on the pyramidal region $\mathcal{D}_{C_{1}}$ (a similar result holds on the other pyramidal regions).

TheOREM 2.4. Let $f_{\text {loc }}$ be the surface fragment given by expression (12) and $\left\{\psi_{j, \ell, k}\right\}$ be the Parseval frame of shearlets for $L^{2}\left(\mathcal{D}_{C_{1}}\right)^{\vee}$ given by (6). Then, for $-2^{j} \leq \ell_{2} \leq 2^{j}$, the following estimate holds:

$$
\int_{\widehat{\mathbb{R}}^{3}}|\hat{f}(\xi)|^{2}\left|\hat{\psi}_{j, \ell, k}(\xi)\right|^{2} d \xi \leq C 2^{-9 j}\left(1+\left|\ell_{2}\right|\right)^{-5}
$$

We refer to Ref.[13] for the proof of Theorem 2.4 and the complete proof of Theorem 2.3.

\section{CONCLUSION}

We have shown that the shearlet approach extends naturally from the $2 \mathrm{D}$ to the $3 \mathrm{D}$ setting. In fact, we have constructed a Parseval frame of $3 \mathrm{D}$ shearlets for $L^{2}\left(\mathbb{R}^{3}\right)$ whose elements are well localized and form a collection of highly anisotropic waveforms ranging over various scales, directions and locations. Thanks to their properties, the Parseval frames of 3D shearlets provide nearly optimally sparse approximations for a large class of piecewise smooth 3D data. This is the first and, so far, the only result of this type, and it is a significant improvement over wavelets and other traditional (nonadaptive) representations, which are very inefficient at approximating distributed discontinuities in multidimensional data.

As we have discussed above, the excellent approximation properties of 3D shearlets are a direct consequence of to their ability to sparsely approximate anisotropic features. In fact, in a related investigation, the authors have proved that $3 \mathrm{D}$ shearlets can be used to precisely characterize the surface of discontinuities of 3D data through the asymptotic decay of the shearlet transform at fine scales. ${ }^{12}$ This result is consistent with similar observations made by the authors and other collaborators ${ }^{10,22}$ in dimension 2 and which have been applied to develop very competitive algorithms for edge analysis and detection.

In fact, it is expected that the theoretical results presented in this work will lead to innovative algorithms for the efficient analysis and processing of 3D data sets, including applications to denoising and feature extraction. In particular, a numerical implementation of the 3D shearlet transform was recently presented by one of the authors with another collaborator, and its application to video denoising was found very competitive. ${ }^{21}$

\section{ACKNOWLEDGMENTS}

The authors acknowledge support from NSF grant DMS 1008900/1008907; D.L. also acknowledges support from NSF grant DMS 0746778.

\section{REFERENCES}

[1] E. J. Candès, L. Demanet, D. L. Donoho and L. Ying, Fast discrete curvelet transforms, Multiscale Model. Simul., 5 (2005), 861-899.

[2] E. J. Candès and D. L. Donoho, New tight frames of curvelets and optimal representations of objects with $C^{2}$ singularities, Comm. Pure Appl. Math., 57 (2004), 219-266. 
[3] F. Colonna, G. Easley, K. Guo, and D. Labate, Radon Transform Inversion using the Shearlet Representation, Appl. Comput. Harmon. Anal. 29(2)2 (2010), 232-250.

[4] D. L. Donoho, Sparse components of images and optimal atomic decomposition, Constr. Approx. 17(2001), 353-382.

[5] D. L. Donoho and G. Kutyniok. Microlocal Analysis of the Geometric Separation Problem, preprint 2010.

[6] D. L. Donoho, M. Vetterli, R. A. DeVore, and I. Daubechies, Data compression and harmonic analysis, IEEE Trans. Inform. Th. 44(1998), 2435-2476.

[7] G. R. Easley, D. Labate, F. Colonna, Shearlet-Based Total Variation Diffusion for Denoising, IEEE Trans. Image Proc. 18 (2) (2009), 260-268.

[8] G. R. Easley, D. Labate, and W. Lim, Sparse Directional Image Representations using the Discrete Shearlet Transform, Appl. Comput. Harmon. Anal., 25 (1) (2008), 25-46.

[9] K. Guo, D. Labate, Optimally Sparse Multidimensional Representation using Shearlets, SIAM J. Math. Anal., 9 (2007), 298-318

[10] K. Guo, and D. Labate, Characterization and analysis of edges using the continuous shearlet transform, SIAM J. Imag. Sci. 2 (2009), 959-986.

[11] K. Guo, and D. Labate, Optimally Sparse 3D Approximations using Shearlet Representations, Electronic Research Announcements in Mathematical Sciences, 17 (2010), 126-138.

[12] K. Guo, and D. Labate, Analysis and Detection of Surface Discontinuities using the 3D Continuous Shearlet Transform, Appl. Comput. Harmon. Anal. 30 (2011), 231-242.

[13] K. Guo, and D. Labate, Optimally Sparse Representations of 3D Data with C2 Surface Singularities using Parseval Frames of Shearlets, preprint, (2010).

[14] K. Guo, W.-Q Lim, D. Labate, G. Weiss and E. Wilson, Wavelets with composite dilations, Electron. Res. Announc. Amer. Math. Soc., 10 (2004), 78-87

[15] K. Guo, W-Q. Lim, D. Labate, G. Weiss and E. Wilson, Wavelets with composite dilations and their MRA properties, Appl. Computat. Harmon. Anal. 20 (2006), 231-249.

[16] G. Kutyniok and W.-Q Lim, Compactly Supported Shearlets are Optimally Sparse, preprint (2010)

[17] J. Lemvig, Optimally Sparse Approximations of Functions in $L^{2}\left(\mathbb{R}^{3}\right)$ with $C^{\alpha}$ Singularities using Shearlet, Oberwolfach Report 44 (2010)

[18] W. Lim, The Discrete Shearlet Transform: A New Directional Transform and Compactly Supported Shearlet Frames, IEEE Trans. Image Process 19(5) (2010), 1166-1180.

[19] S. Mallat, A Wavelet Tour of Signal Processing.Third Edition: The Sparse Way, Academic Press, San Diego, CA, 2008.

[20] F. Natterer, and F. Wübbeling, Mathematical Methods in Image Reconstruction, SIAM Monographs on Mathematical Modeling and Computation, Philadelphia, 2001.

[21] P. Negi and D. Labate, 3D Discrete Shearlet Transform and Video Denoising, Poster presentation at FFT Talks 2011.

[22] S. Yi, D. Labate, G. R. Easley, and H. Krim, A Shearlet approach to Edge Analysis and Detection, IEEE Trans. Image Process 18(5) (2009), 929-941. 